\title{
Filigrane
}

Écoutes psychothérapiques

\section{"Mort ou faute » : essai sur les fantasmes de suicide-limite comme effets d'une différenciation interdite}

\section{Anthony Bourgeault}

Volume 15, numéro 1, printemps 2006

URI : https://id.erudit.org/iderudit/013532ar

DOI : https://doi.org/10.7202/013532ar

Aller au sommaire du numéro

Éditeur(s)

Revue Santé mentale au Québec

ISSN

1192-1412 (imprimé)

1911-4656 (numérique)

Découvrir la revue

Citer cet article

Bourgeault, A. (2006). « Mort ou faute » : essai sur les fantasmes de suicide-limite comme effets d'une différenciation interdite. Filigrane, 15(1), 99-112. https://doi.org/10.7202/013532ar
Résumé de l'article

À partir d'une lecture de La Métamorphose de Franz Kafka, l'auteur propose une théorie du suicide-limite qui tienne compte de la pathologie familiale introjectée par le sujet suicidant. D'abord, la transformation radicale de Gregor Samsa en cancrelat et, ensuite, sa lente agonie vers la mort seront comprises comme ses tentatives de se différencier d'une famille aux limites mal régulées. Paradoxalement, le passage à trépas de Gregor sera aussi sa seule façon de se réunir avec une image idéalisée de sa famille, ébranlée par sa récente métamorphose. L'article permet également de discuter de l'étrangeté mortifère qui envahit l'expérience-limite du corps et du langage, enferme le sujet dans une solitude sans bons objets intériorisés, et le fait glisser vers la mort, devenue l'ultime solution à son problème d'exister. 


\title{
«Mort ou faute »: essai sur les fantasmes de suicide-limite comme effets d'une différenciation interdite
}

\author{
anthony bourgeault
}

\begin{abstract}
À partir d'une lecture de La Métamorphose de Franz Kafka, l'auteur propose une théorie du suicide-limite qui tienne compte de la pathologie familiale introjectée par le sujet suicidant. D'abord, la transformation radicale de Gregor Samsa en cancrelat et, ensuite, sa lente agonie vers la mort seront comprises comme ses tentatives de se différencier d'une famille aux limites mal régulées. Paradoxalement, le passage à trépas de Gregor sera aussi sa seule façon de se réunir avec une image idéalisée de sa famille, ébranlée par sa récente métamorphose. L'article permet également de discuter de l'étrangeté mortifère qui envahit l'expérience-limite du corps et du langage, enferme le sujet dans une solitude sans bons objets intériorisés, et le fait glisser vers la mort, devenue l'ultime solution à son problème d'exister.
\end{abstract}

«On ne se tue jamais que pour exister.»

André Malraux

$\mathrm{D}$ ilemme précédant le suicide : mort ou faute. Le titre choisi pour cet essai repose l'antagonisme entre la vie et la mort dans la perspective d'un suicidant-limite: dans son expérience, la faute se substitue à la vie (motemblème d'une subjectivité désirante), ses désirs de sujet lui sont barrés parce que son authentique différenciation est interdite. Il ne peut plus alors que mourir... ou plus exactement, que désirer mourir. Est-ce donc à dire qu'il lui reste encore de ses désirs ? Mort... Et faute. Le fantasme suicidaire est paradoxal : il révèle toute l'impossibilité du sujet à désirer vers la vie autant qu'il ébauche l'ultime manifestation de son désir, il vise la fusion avec un objet idéal qui le sauverait de ses manques (et par là qui lui éviterait l'épreuve de désirer) autant qu'il cherche à établir la marque de sa différence désirante par rapport à son monde objectal envahissant. Le suicide apparaît alors comme une dernière faute existentielle, comme le fatum d'une mésaventure mortifère de la subjectivation. Dans ce sens, le suicide commet ce que le sujet ne peut commettre autrement: quelque chose de son désir (cette faute qui produit sur l'Autre parental un effet d'horreur).

Cette horreur paniquée pour la Chose qui inscrit l'autre dans son altérité, pour cette différence qui rend le familier étranger, est vécue par les parents de Gregor 
Samsa lorsque celui-ci se transforme en cancrelat dans La Métamorphose de Franz Kafka. Cette métamorphose fracture l'uniformité de la famille et l'installe dans une crise qui se résoudra par la mort de Gregor: l'absence de ce fils fait étranger permettra à la famille de se ressouder pathologiquement, de renier l'intersubjectivité et la séparation qui la fonde.

À l'origine, l'audace de cet essai était double : articuler la théorie clinique de la personnalité-limite suicidaire à une nouvelle de fiction, et surtout, penser la nouvelle de Kafka comme une métaphore d'un suicide (alors que personne ne s'y suicide au sens très premier du terme). Mais n'est-ce pas la prétention de l'écoute (de la lecture) analytique que d'entendre entre les lignes, que de rêver à partir d'un matériel concret pour le transporter dans un ailleurs de sens, que de restituer le désir qui se dérobe à la mise en scène manifeste - ici le désir suicidaire ? En interprétant l'intrigue de la nouvelle comme celle d'un suicide, j'avais cependant l'impression de la dénaturer exagérément. Il manque à Gregor un passage à l'acte létal pour qu'il y ait véritablement suicide. Il y a désir de mourir mais pas de geste qui le réalise : la mort pénètre (dans un coït fatal, une scène finitive) un Gregor passif qui l'attend. Dans cet ordre d'idées, l'essai porte désormais sur le fantasme de se suicider plutôt que sur le suicide en tant que tel.

Ma tentative est celle de réfléchir sur la fonction duelle - fusionnante et radicalement individuante — du fantasme suicidaire, cohérente avec l'impasse des sujets de personnalité-limite. Effectivement, ceux-ci apparaissent prisonniers d'une transitionnalité trouble dans laquelle sujet et objet assument difficilement le paradoxe d'une séparation et d'une réunion concomitantes. Afin de comprendre comment ce fantasme peut en venir à s'implanter dans la psyché-limite, je proposerai d'abord une genèse relationnelle de cette organisation de la personnalité que je situerai ensuite par rapport aux enjeux subjectifs de l'adolescence. La thèse que je défendrai propose que le fantasme de suicide apparaît comme solution à un destin traversé d'un interdit préoedipien de différenciation. Dans ma démarche, je suis accompagné de la famille Samsa, cause de l'essai, dont je cherche à élucider la folie privée en y insufflant un peu du sens psychanalytique, en nommant autrement ses conflits de subjectivation.

\section{Il aurait été une fois... le traumatisme des limites}

La métamorphose survient à un moment vaguement circonscrit dans la vie de Gregor, à l'orée de sa jeunesse d'adulte. Quelle préhistoire imaginée à cette nouvelle ? Sûrement celle de la petite enfance associée à la personnalité-limite et à ses traumatismes compromettant l'achèvement heureux de la première phase d'individuation-séparation.

Raymond Cahn (1998) parle de l'état-limite (dont le borderline fait partie) comme d'un «état qu'il serait pertinent de considérer comme limite, non entre névrose et psychose, mais entre capacité et incapacité à accéder à la position de sujet» (p. 54). Cette condition de presque sujet s'organise sous l'influence d'objets hyperexcitants et hostiles qui renvoient à une mère (ou à ce qui en a fait figure) dont les trop d'intrusion ou d'absence ont fait sur l'enfant effet de violence. 
À un niveau physique, ces excès et manques de manque compromettent la définition d'une limite distinguant le dedans du dehors. La jouissance du corps n'est alors pas médiatisée et connote insuffisamment le passage d'une nourriture extérieure vers l'intérieur (Painchaud et Mongrain, 1986): ou précocement gavé, le nourrisson rate son expérience de désirer et se trouve aliéné à sa satisfaction, ou exagérément frustré, il est abandonné à son avidité désorganisante qui menace son sentiment d'existence continue. Selon une antinomie comparable, Masterson (1975, 1987) comprend la dynamique relationnelle de la dyade-limite mère-enfant en termes de disponibilité et d'indisponibilité libidinales de la mère : s'opposant à l'autonomisation de son enfant, soit elle le comble de soins lorsque celui-ci se montre docilement passif, soit elle les lui retire lorsqu'il s'exprime d'une façon autre que celle prescrite par son désir maternel infantilisant. Conséquemment, l'enfant vit des épisodes contradictoires : tantôt il se soumet à sa mère de laquelle il dépend afin de fuir une angoisse douloureuse d'abandon, tantôt il se manifeste dans ce qui est perçu comme une révolte irrecevable et punie par la mère. Il sombre alors dans l'instabilité de son devenir, dans une angoisse de n'être plus rien pour elle. Narcissiquement vulnérabilisé, sa rage orale est ce qui lui reste d'actif pour se vivre comme différent, pour éviter la néantisation d'une mort psychique. Shapiro et al. (1975) complètent les propositions théoriques de Masterson: bien qu'ils reconnaissent que certains parents d'états-limites désapprouvent l'autonomie de leur enfant, celle-ci leur apparaissant comme une condamnation hostile de leurs bons soins, ils soulignent que d'autres parents désapprouvent hargneusement sa dépendance, comprise comme expression d'exigences capricieuses. La prévalence de l'un ou l'autre des scénarios dépend du rapport d'identifications projectives que ces parents entretiennent avec leur enfant: nient-ils plutôt, pour le projeter et le condamner ensuite, leur propre besoin de dépendance ou leur propre souhait d'autonomie?

Cette maternité (voire cette parentalité) vécue dans les extrêmes témoigne de l'incapacité parentale à reconnaître l'identité différente de leur enfant. L'enfantlimite devient l'objet-écran, narcissiquement investi et contrôlé, des identifications projectives des parents qui y inscrivent leurs représentations clivées d'eux-mêmes (ou de leurs propres objets parentaux). L'anaclitisme attendu de la relation est inversé : c'est le parent qui fait reposer sur son enfant la responsabilité de préserver son équilibre. Ainsi assimilé au narcissisme parental et insuffisamment aimé pour lui-même, l'enfant corporel et psychique est sacrifié à un fantasme létal d'indifférenciation qui s'accomplit d'abord dans l'inconscient de ses parents (Manzano et al., 1999) et à partir duquel son monde interne se constitue en partie. Josef Perelberg (2004) parle d'ailleurs de meurtre d'âme pour désigner cette tentative parentale de mettre en péril l'identité séparée, soit la capacité désirante, d'autrui.

Privé d'un regard sur ce qui le différencie de l'enfant imaginaire destiné au Moi Idéal des parents, l'enfant-limite subit la violence narcissique (le vide spéculaire) de la quête du même de ses géniteurs : il apprend qu'il doit être autre que ce qu'il est, qu'il doit être faux ou n'être rien (Wainrib, 1996). La perception se substitue 
trop rapidement à l'aperception (Winnicott, 1975): tache aveugle dans le champ visuel de l'Autre, l'enfant-limite est alors celui qui doit voir pour aller récolter l'amour - qui ne lui est pas donné inconditionnellement. Dans l'absence d'une fonction réflexive symbolique, l'enfant-limite est accablé du poids d'un ensemble d'états mentaux, étrangers et hostiles, qui se logent dans la structure de son self alors qu'ils appartiennent à l'expérience de l'Autre (Fonagy, 2004). Le self est contaminé : il est opprimé par la représentation d'un Autre avant que ses limites n'aient été suffisamment formées. La personnalité-limite correspond donc à un état de la difformité des limites, fendues de manière persécutante par l'étrangeté de corps intrusifs.

Construite dans un rapport d'étrangeté importune à elle-même, la psyché-limite lutte ainsi contre son anéantissement : elle reste fixée dans l'impuissance originaire de l'insatisfaction prolongée, ses premiers désirs ayant été négligés par un objet aliénant qui y a massivement superposé les siens. Les débordements agressifs spontanés du nourrisson ne sont pas contenus par les rêveries d'une mère apaisante, ils sont plutôt doublés d'une submersion séductrice et hostile par les rêveries maternelles transformées en cauchemars. La mère ne survit pas aux tentatives de destruction de son enfant mais contre-attaque par un abandon foudroyant de celuici ou par des sévices contre sa vivacité : par suite, l'extériorité de l'objet — son droit à l'indépendance et au secret — est mitigée, le dehors appartient encore au dedans (Winnicott, 1975), la fatalité de la séparation est indéfiniment (infiniment) ajournée. Manquant à être soutenu, le développement affectif du self est ainsi compromis. Puisque la mère n'a pas toléré l'ambivalence de son enfant en cherchant à lui interdire une partie de sa subjectivité, le monde interne de ce dernier est clivé : il est occupé par des objets persécuteurs, unilatéralement mauvais, dont la menace est contrecarrée par leur idéalisation sporadique (Masterson, 1975).

Cet empiétement meurtrier de l'environnement sur le nourrisson interdit tout espace de repos à son expérience individuelle. Son individualité est donc imbriquée à celle de la mère envahissante et son activité pulsionnelle est vécue comme réaction aux exigences du monde externe (Winnicott, 1955). Il ne peut donc exister qu'en extension soumise aux désirs de l'Autre. Il est ainsi contraint de décentrer sa position désirante, d'abdiquer à ses désirs et de les remettre dans le lieu de l'Autre: il s'adapte ainsi à un Autre qui veut à sa place et qui lui en veut d'être différent. Subséquemment, l'enfant-limite identifie difficilement ses propres pensées, sentiments et désirs. Il est perdu derrière un faux self, éprouvé comme vrai, qu'il s'est construit comme carapace aux aléas de l'amour destinée à combattre son individuation et l'angoisse de séparation qui l'accompagne (Masterson, 1987). De cette façon, il reste accroché aux demandes aliénantes de l'objet et se protège d'une exploitation de son vrai self qui, s'il était exposé, risquerait l'anéantissement (Winnicott, 1974) - anéantissement dont la menace est audible en sourdine dans les expériences-limites.

Sa capacité à être seul, à comprendre comme capacité à être seul devant du différent reconnu et utilisé comme tel, se retrouve ainsi endommagée. Sa mère ne 
s'étant pas suffisamment laissée investir par les gestes spontanés de son enfant, celui-ci a donc dû essuyer des ruptures dans l'expérience de ses pulsions comme réelles (Winnicott, 1958). Conséquemment, il ne peut saisir ce vrai self parce qu'il est privé de la pleine jouissance des fonctions corporelles sur lesquelles il s'étaie. Inauguré par une connaissance non pensée, le vrai self ne se découvre que dans l'existence (Bollas, 1996), que dans une relation à un monde qui le laisse être, que dans un rapport actif d'intentionnalité avec un extérieur réceptif. L'enfant-limite méconnaît donc sa vie personnelle parce que son monde objectif à lui échoue à accueillir son altérité dynamique. La mère insuffisamment bonne refuse la solitude existentielle de son enfant - qui sous-tend l'unicité de son expérience désirante — de façon à nier la radicalité de leur différence qui la renvoie à sa solitude à elle, insoutenable.

L'inertie de la fonction paternelle, inductrice de sens et moteur dans le processus de subjectivation, contribue également à l'incapacité à être seul de l'enfant-limite: en effet, n'intervenant que trop peu, le père (ou celui devant s'acquitter de sa fonction) lâche l'enfant dans le piège sadomasochiste maternel (Campbell, 2004) et dans le vide psychique qui s'ensuivra. Il ne freine donc pas l'immixtion de la mère dans l'univers interne (les relations d'objet intériorisées, les fantasmes) de l'enfant. L'absence du père fait donc effet de traumatisme négatif (Magnenat Bourquin, 1995) qui laisse vide la case tierce du bon à symboliser et irrésolue l'impasse de la séparation inachevée (car interdite par la mère et conséquemment intolérable pour l'enfant). Même dans ses intermittentes présences, le père est relégué au silence de son trou noir. En effet, narcissiquement dépouillé parce qu'ayant dû donner à la vie alors qu'il était dans l'ombre de la mort (Paz et Olmos Paz, 1992), l'enfant-limite affronte avec rage son exclusion de la scène primitive qui le précipite dans le réel de l'absence, la mort (Jeammet et al, 1994). Conséquemment, il la dénie par son omnipotente illusion à pouvoir réaliser tous les fantasmes et attentes de la mère (Schachter, 2004).

En conséquence, l'enfant-limite ne parvient à élaborer qu'une aire antitransitionnelle dans laquelle le paradoxe de l'objet trouvé-créé est insupportable. Effectivement, la coïncidence dans un même espace expérientiel d'une séparation et d'une réunion avec un symbole de mère est antithétique avec les pratiques maternelles, celles-ci manquant de cette nuance intersubjective selon laquelle deux sujets peuvent être ensemble mais séparés, parents mais distingués. Chez l'enfantlimite, la transitionnalité du paradoxe séparation-réunion prend plutôt la forme d'un drame à la bipolarité radicale, que je nomme abandon-indifférenciation, rendant difficile l'aménagement d'une intermédiateté créatrice. En effet, sa mère lui usurpe son aire de jeu : elle y injecte son propre matériel qui finit par le persécuter parce qu'il n'arrive guère à le rejeter. L'ascendant maternel et ses intentions destructrices se superposent à ce qui aurait pu devenir sa liberté symbolisante. Il lui est donc interdit de s'individualiser par un jeu créateur: au risque de perdre la mère, sa libido ne peut guère s'égarer de cette dernière ou du cadre que celle-ci a rigidement délimité. Ainsi paralysé par l'étrangeté de l'Autre qui s'incruste en lui, 
il lui manque la sécurité pour émanciper sa créativité. Écrasé par le monde extérieur, il est donc coupé de sa vivacité interne et a tendance à agir sans vie subjective vraie. Son fonctionnement mental se clivant de la vérité de ses désirs, il s'enferme graduellement dans un leurre existentiel au sein duquel il déforme ses réalités intra et extra psychiques (Cahn, 1988).

Ainsi, la personnalité-limite se pense d'abord comme objet du désir d'un Autre incestuel et meurtrier (Richard, 2001) et non comme sujet désirant. Enfermé dans une dialectique relationnelle tyrannique, la psyché-limite a dû retrancher ses désirs désavoués par ses premiers objets à la doctrine du Tout ou Rien («Sois ce qu'il me faut ou ne soit pas»). Le self-limite persévère ainsi dans le faux et les duels aliénants, sous un impératif d'emprise, plutôt que dans le vrai et la reconnaissance triangulée de la différence.

\section{Adolescence, l'étrange et la redéfinition des limites...}

La puberté, par la poussée pulsionnelle qui la caractérise, donne à l'enfantlimite une seconde chance pour habiter pleinement une position de sujet désirant, assumée comme telle, à la condition que se manifeste la fonction paternelle qui la fera respecter. Effectivement, la puberté est causée par la pulsion qui cherche à s'affirmer. Matrice originaire sur laquelle se fondent la subjectivité et le sentiment d'être séparé de l'objet (Richard, 2001), la pulsion pubertaire — par sa force et sa nouveauté - rend possible l'expérience du self psychique et corporel comme vraie, selon l'accueil qui lui est réservé par l'environnement du pubère en éveil. Elle précipite ainsi le prétendant sujet dans une seconde phase d'invididuationséparation, dans le travail psychologique de l'adolescence rendu nécessaire par les transformations physiologiques spontanées. Ce processus est toutefois distinct d'un âge fixé dans l'histoire du sujet mais constitue plutôt une structure évolutive au sein de laquelle celui-ci a à se dégager des objets de son passé (Richard, 1998). Le traumatisme de l'éruption génitale du corps provoque ainsi une crise - dont l'étymologie désigne «un moment d'hésitation suivi d'une décision» i.e. un moment de subjectivation - à la résolution parfois indéfiniment reportée, voire interminable: c'est le cas de la personnalité-limite, alors située entre adolescence et psychose (Richard, 2001).

Le défi périlleux de l'adolescent consiste en cela qu'il a à supporter les tensions générées par l'oxymore ressenti du familier étranger: la maturation sexuelle de son corps sert effectivement de vecteur à la résurgence d'une sexualité infantile déjà connue mais jamais vécue, au télescopage des désirs incestueux et parricides (Cahn, 1991). Je dirais même que son véritable défi identitaire est aussi celui de renverser ces connotations de familier et d'étranger: d'une part, il doit se familiariser avec son corps étrange, d'autre part, il doit s'étranger par rapport à ses objets familiaux (Le Run, 1999).

Selon cet ordre d'idées, l'adolescent doit vivre son corps pubère comme réel et être reconnu dans sa nouvelle image — porteuse de ses désirs émergents — plus facilement assimilable si elle lui est positivement redonnée à voir. Pour ce faire, 
son entourage doit le laisser être ainsi en irruption (i.e. immature, impulsif et désorganisé selon des critères adultes), de façon à renforcer sa créativité et son individualité en probation (Winnicott, 1962).

Également, la valorisation de sa créativité permet à l'adolescent de rejeter ses parents, de les mettre symboliquement à mort (et ceux-ci ont à survivre réellement à leur assassinat), de leur causer un manque grâce auquel il peut exister en les déidéalisant: les objets archaïques sont alors substitués par de nouveaux objets d'investissement qu'il choisit. L'adolescent-sujet renaîtra donc des suites d'un travail de négativation opéré sur l'objet: ce dernier doit être perdu, expulsé partiellement de la psyché pour être établi en position d'altérité. Se creuse ainsi une béance subjectivante, un espace d'absence au creux duquel un je peut advenir et désirer (Richard, 2001). L'aventure de la subjectivation implique donc une prise de conscience de la séparation avec le monde (de la mère et du père) éprouvé comme Autre, un désengagement et une désaliénation par rapport à son emprise. Le je s'émancipe alors dans la dépression induite par un sentiment de perte (objectale, idéale), conditionnelle pour l'élaboration d'un univers interne doué de pensées et d'affects reconnus comme siens. C'est grâce à cet espace foré par le je que le sujet s'approprie son corps sexué, contient ses deuils de Moi Idéal et de jouissance maternelle, s'organise et donne sens aux incessants changements qui le font autre en même temps qu'il demeure lui-même (Cahn, 1998). L'accomplissement de la différenciation à l'adolescence ouvre donc le sujet sur l'appropriation de sa réalité psychique, sur la mise en sens de son expérience du monde et ultimement sur l'adéquation de son être physique avec un sens métaphysique, i.e. de son acte avec sa pensée.

L'adolescent-limite échoue cependant à réaliser son identité séparée et manque à profiter de son gain de sens. Ses parents s'opposent au rythme progressif et concluant d'un parcours vers son individuation : ou bien ils disparaissent précocement comme repères fiables ou bien ils s'imposent oppressivement dans son champ expérientiel qui ne lui appartient plus. L'adolescent-limite apparaît donc comme territoire déserté ou colonisé par l'empire parental. Il est ainsi dépourvu d'objets fixes, de points d'ancrages anaclitiques, pour représenter la constance dans sa subjectivité en mutation et ainsi contenir la désorganisation résultant de sa crise identitaire (Paz et Olmos Paz, 1992).

Les carences de la fonction paternelle, qui aurait mené à la création de limites pare-excitantes dans l'archaïsme reviviscent de la relation mère-enfant (Josef Perelberg, 2004) et qui aurait ainsi favorisé l'espace nécessaire au bon déroulement des phénomènes de la transitionnalité, laissent l'adolescent-limite dans le vif d'une tension antitransitionnelle entre crainte et désir de dépendance (Ottino, 1995). En effet, il redoute l'Autre, de par sa charge envahissante qui le pousse aux bords de l'explosion pulsionnelle, en même temps qu'il le recherche, trop vulnérable dans l'expérience d'une solitude dans laquelle il risque de se perdre, n'ayant appris à exister qu'en réaction aux injections psychiques de cet Autre. En conséquence à cette dialectique de la dépendance, il oscille entre des sentiments 
de vide contrecarrés par une certaine omnipotence qui le remplit en illusions (Richard, 2001).

Ainsi, l'adolescent-limite échoue à se familiariser avec sa subjectivité, à créer une béance suffisamment ouverte à ses désirs, puisqu'il n'arrive à se dégager de cet Autre intrusif, cause de son étrangeté, institué au cœur de son être. Sous l'emprise de l'objet et submergé de ses pulsions, il procède mal à la tâche adolescente de différenciation-désaliénation. Ses mécanismes de défense de clivage, de déni, d'identification projective, visant à le protéger de ses conflits, l'empêchent d'en être le sujet: ceux-ci sont plutôt éjectés hors de sa psyché (Cahn, 1998). La somatisation et l'acte priment sur le sens. L'enfer, c'est celui du je qui s'interdit au lieu d'advenir (parce que l'enfer, c'était d'abord l'Autre) ${ }^{1}$.

\section{Illustration littéraire : l'adolescent-parasite ${ }^{2}$}

Il s'agit maintenant d'illustrer allégoriquement ce vécu-limite traversé de l'interdit de différenciation. Cette vignette clinico-littéraire me servira de tremplin à la présentation d'une solution possible à l'expérience de cet interdit : le fantasme suicidaire.

Le destin de Gregor pose la question du comment être soi, avec tous les paradoxes qu'elle comporte. Jeune adulte, il semble vivre normalement les défis d'une adolescence prolongée : il cherche à élaborer une distance par rapport à ses parents tout en ne les perdant pas. Ainsi, bien qu'il soit voyageur de commerce, il habite encore avec eux et sa sœur (il barre toutefois la porte de sa chambre). Ses difficultés à se différencier de l'Autre parental apparaissent d'emblée sous un angle subtil : il voyage et par là se sépare, mais pour le compte de ses parents qui ne travaillent pas, pour rembourser une dette du père. Son autonomie est ainsi développée pour soutenir ses parents de qui il devient l'objet anaclitique. Pour être aimé, il doit se conformer au désir parental qu'il les fasse vivre (qui est là pour le laisser vivre lui ?). Il est pris dans une identification projective de ses parents pour qui il représente un objet parental idéalisé, merveilleusement pourvoyeur. Premier indice d'une relation aliénante.

Le drame se déclare le matin de la métamorphose de Gregor: «il se retrouve dans son lit changé en un énorme cancrelat» (p. 79). Ça l'a changé, ajouterais-je, soit la dualité de ses désirs (sur l'axe fusion-individuation radicale ou encore sur l'axe de la dépendance voulue-crainte) innommés mais évacués par une somatisation monstre, d'un extrémisme corrélatif à sa détresse non symbolisée. La métamorphose étaie un mouvement double de désubjectivation et de resubjectivation.

D'une part, elle vise à renverser le parasitisme psychique et financier dont les parents de Gregor tiraient profit jusqu'à maintenant, à inverser la dépendance anaclitique: transmuté en coquerelle, il tente de satisfaire son besoin de dépendance envers ses parents désormais responsables de sa survie. Il se condamne (parce qu'il a déjà été condamné) à ne plus les quitter. Sa métamorphose est alors demande refusée d'amour, appel sans réponse à l'Autre, supplication rabrouée d'abolir les barrières qui le séparent de ses parents en s'enfermant éternellement 
avec eux dans un même appartement. D'ailleurs, jamais dans la nouvelle ne faiton référence à une promenade au dehors de l'édifice parental ou à une porte pouvant y mener (Thiébault, 1991). D'autre part, sa métamorphose frappe avec la violence d'une révolte, dirigée contre un Autre qui le chosifie. Dans le même sens, elle représente une fuite vers une subjectivité radicalement différente, au-delà d'une soumission rendue impossible (Deleuze et Guattari, 1975). Concrétisant le rejet des identifications à sa race, à sa culture voire jusqu'à sa langue, elle met en corps sa lutte adolescente pour se sentir réel, pour établir une identité indépendante qui n'appartienne qu'à lui.

Le nouveau corps de Grégor est aussi un corps qui mue et qui annonce par une antipuberté que rien ne pourra être comme avant. Il ne pourra plus travailler, incapacité par laquelle il se différencie autant qu'il régresse. Il ne mangera plus la nourriture qu'il mangeait la veille de sa transformation mais se satisfera d'une pitance plus primitive, en décomposition. Son corps est lieu de son trouble, de son inquiétante étrangeté, du retour d'une pulsionnalité sauvage qui le tire vers les plaisirs prégénitaux infantiles dans la reddition à une mère archaïque. Cependant, ce familier fait un retour dans une consistance radicalement étrange, comme si ce familier ne lui avait jamais été vraiment familier, comme si Gregor avait toujours été étranger à son expérience pulsionnelle, comme si celle-ci n'avait jamais été qu'une affaire de l'Autre. Il réagit donc avec peu de surprise à sa métamorphose, laissant ainsi à penser qu'il y reconnaît là une expérience pour lui habituelle, celle de ne pas se reconnaître : c'est plutôt son étrangeté — l'expérience d'être étranger à soi — qui lui serait familière.

Ses parents ne survivent pas à l'agressivité individualisante de la métamorphose pas plus qu'ils ne s'acquittent amoureusement de leur rôle nourricier. Au contraire, ils condamnent sévèrement Gregor qui a échappé à sa fonction anaclitique. L'altérité du fils est menaçante: elle est vécue comme une trahison. Ils ne soutiennent donc son étrangeté d'aucun regard bienveillant: ils ne reflètent que leur terreur devant la Chose. La métamorphose de Gregor tue presque sa mère lorsque celle-ci ose le regarder: son élan vers le différencié est une faute, un crime. Ancien objet idéal de ses parents, il devient leur objet persécuteur soumis à la loi du Talion au sens où ils le persécutent à leur tour. Ils s'élèvent ainsi contre sa différenciation qui fend la masse familiale uniforme parce qu'elle force chacun de ses membres à s'autonomiser, à travailler — étant donné «la nouvelle nécessité à gagner de l'argent» (p. 111).

Abhorrant l'étrangeté de leur fils, ils ne peuvent se l'approprier macabrement qu'en la colonisant de leurs projections hostiles : le père, «résolu à le bombarder » (p. 124), blesse ainsi Gregor en le tirant d'une pomme qui restera encastrée dans sa carapace d'insecte. Ce geste meurtrier matérialise bien la constitution d'un selflimite étranger à lui-même parce que réceptacle d'introjects hostiles, imposés précocement par un Autre dont la violence attaque la différenciation. «On ne le comprend pas » (p. 106), on l'agresse plutôt. La chambre de Gregor est également vidée du mobilier qui lui appartenaient pour être convertie en salle de débarras, 
«on lui prend ce à quoi il tient» (p. 119) pour le remplacer par «des objets qu'on ne peut mettre ailleurs » (p. 132) : l'espace de Gregor est alors envahi de toutes les choses usées dont ses parents ne veulent plus (définition métaphorique de l'identification projective: ce que la psyché ne peut plus supporter, elle le déporte sur celle d'un Autre qu'elle contrôle en l'assimilant à elle-même). De cette façon, ils ne rencontrent pas la différence de leur fils mais l'utilisent plutôt pour étendre leur propre territoire. «Il ne lui reste plus aucune place pour bouger» (p. 133). Gregor est alors coincé «dans l'ombre» (p. 103) de ces objets étrangers qui l'immobilisent dans l'aire d'un jeu spontané, désormais impossible.

Dans ce sens, la famille Samsa ne tolère pas le paradoxe transitionnel de la séparation-réunion. Transformé en coquerelle, Gregor découvre autrement ses meubles humains, comparables à des objets créés-trouvés: le canapé déjà là devient pour lui un lit, le plafond est utilisé comme piste acrobatique. Cependant, sa famille le prive de toute expérience créative en reterritorialisant ainsi ce qui était sa pièce privée. L'antitransitionnalité y est plutôt envisagée sous l'angle-limite de l'abandon-indifférenciation. L'utilisation de la porte de chambre de Gregor dans l'appartement révèle d'ailleurs une différenciation mal régulée, une famille qui ne respecte pas la distance séparant-unissant deux sujets différents. Ainsi, cette porte se barre mais menace d'être défoncée (la porte qui sépare stimule un mouvement d'indifférenciation - la fonction paternelle faillit) ou bien elle s'ouvre mais on exige de Gregor qu'il reste caché (la porte qui réunit contraint à l'abandon - la fonction maternelle aguiche et déçoit). «Le matin, lorsque toutes les portes étaient fermées, tout le monde avait voulu entrer et maintenant qu'il avait lui-même ouvert l'une des portes, personne ne venait et on avait mis les clefs à l'extérieur» (p. 103). Gregor est d'ailleurs de plus en plus impuissant dans toute cette fluctuation des limites: l'Autre régule à sa place — nonobstant ses désirs — la valse des portes, les allers et venues sur son territoire aliéné, les échanges entre le dehors et le dedans brouillés.

Gregor vit alors sa métamorphose dans une solitude vide de bons objets intériorisés : ceux à qui il pense «se détournent tous de lui» (p. 129). Typiquement limites, ses représentations de lui-même et de l'Autre sont clivées. Ainsi oscillet-il entre des creux asthéniques lors desquels il ne peut que rester étendu, à périr, et des glorifications omnipotentes qui l'illusionnent sur sa capacité «à reprendre en main les affaires de la famille» (p. 129). Également, traverse-t-il quelques épisodes de rage - il est alors «dressé de tout son haut, dans une position bien faite pour inspirer la terreur» (p. 113) et ressent sa «fureur à cause du manque de soins » (p. 129) — de la destructivité de laquelle il se défend par une idéalisation prédominante des instances parentales (dont la charge agressive participe probablement à l'économie des identifications projectives de Gregor) qu'il aime dans le déni de leur haine. Ainsi, Gregor est soumis à des relations anaclitiques dans lesquelles les rôles du fort et du faible basculent continuellement, quoique toujours unis dans un même destin de dépendance qui proscrit le dénouement de leur différenciation. 


\section{Aux limites de n'être pas : naître à trépas}

Cette dépendance quasi existentielle de l'adolescent-limite, doublée d'un besoin de contrôle et de possession de l'objet (Samy, 1991), l'enferme donc dans des rapports d'emprise qui permettent un lien de continuité entre les protagonistes, interdits à être différents autant qu'ils sont étrangers à eux-mêmes. L'adolescentlimite cherche donc à contrôler l'Autre dans la mesure où il ne se contrôle pas luimême, débordé par un monde interne auquel il est aliéné, rempli d'affects et d'introjects hostiles (Maltsberger, 2004). Le fantasme de suicide se fonde sur la relation du self à son corps et à ses objets primaires (Campbell, 2004). Il ouvre un espace psychique paradoxal, construit sur l'antitransitionnalité abandonindifférenciation, qui solutionne radicalement cette impasse d'une dépendance insoutenable. Il s'acquitte par là autrement des défis de l'adolescence.

D'un côté, le fantasme de suicide-limite espère retrouver le self en faisant exister la pensée. Il s'agit d'une fantaisie magique qui doit le sauver d'une annihilation psychique (Maltsberger, 2004). La violence qui la scénarise vise à affirmer une distance par rapport à un Autre avide, assimilé au corps de la personnalité-limite, qu'il faut attaquer pour survivre (Bateman, 2004). Il s'agit donc d'un fantasme selon lequel les limites du self se poseraient dans la douleur, dans une tentative de se dégager des objets internes persécuteurs (Fonagy, 2004). Le suicidaire recherche donc à établir une cohérence interne qui lui a toujours été impossible, aliéné à sa subjectivité par la massivité des identifications projectives parentales qui le contrôlent et qu'il cherche à chasser (Kamel, 1988). Son fantasme représente ainsi la survenue d'un sujet grandiose reconnu pour lui-même, différencié d'un idéal parental autocratique (Ribas, 1991b). L'aspiration resubjectivante du fantasme suicidaire tombe cependant dans une mégalomanie délirante qui dénonce l'ordre vital et qui abandonne le monde de l'Autre en niant en avoir besoin. Le fantasme revendique alors l'autosuffisance plutôt que le manque, la maîtrise sur la mort plutôt que l'absurdité du destin, l'omnipotence plutôt que l'acceptation des limites entre le sujet et ses objets (Jeammet et al., 1994). Opérant à la négation de la validité de la scène primitive, le suicidaire-limite se crée pour se détruire dans son fantasme qui en est un d'autoengendrement (Ribas, 1991a) : en décidant de mourir alors qu'il n'a pas décidé de sa vie, il se pose morbidement comme son propre géniteur.

À l'interface de cette illusion d'omnipotence rêvant d'une individuation radicale par la mort, se trouve une estime de soi effondrée, une impuissance qui appelle à la fusion désubjectivante. Ainsi, autant le suicidaire-limite cherche à échapper à une soumission passive à l'objet, autant il est en quête du nirvâna d'un état narcissique indifférencié (Ladame et Ottino, 1993). Dans ce sens, il fantasme se débarrasser d'un corps sexuel qui l'assigne dans les limites d'une incarnation qu'il ne peut assumer (Ladame, 1991). Il souhaite renoncer à ce corps génital qui le définit comme individu séparé - mais dont la séparation est entravée par l'incursion aliénante de l'Autre dans son self — afin de pouvoir renouer avec le temps mythique d'un avant la différence, d'un avant l'étrangéisation. Dans son 
délire suicidaire, une partie de son self survit à la mort corporelle et retrouve une fusion avec la mère idéalisée (Campbell, 2004). Le fantasme de suicide expulse ainsi imaginairement le sujet de la mauvaise mère (ou expulse la mauvaise mère du sujet) en même temps qu'il accomplit son retour incestueux à la madone de l'Edipe archaïque. Le suicidaire-limite prétend à un état d'illimité, à un Rien qui tient lieu de Tout, à un néant où s'indifférencier (Wainrib, 1996). Le désir de mort se représente alors comme un ultime désir avant la fin du désir, comme une expérience limite de solitude avant l'implosion de cette solitude, comme une souffrance extrême avant l'insensibilité à la souffrance. L'enjeu devient celui de savoir combien de temps le suicidaire-limite contiendra l'effet excitant de son fantasme avant de passer à l'acte ou combien de temps il s'écoulera avant que le fantasme lui-même soit ressenti comme un étranger à éradiquer, paradoxalement, par son actualisation (Jeammet et al., 1994).

«L'idée qu'il n'avait plus qu'à disparaître» (p. 143) s'est implantée dans l'esprit de Gregor Samsa. Cependant, il n'accomplit pas lui-même son fantasme de suicide. En dernière instance, la Mort s'en charge alors qu'il est plongé dans un état «de méditation vide et paisible» (p. 143). À quoi pense-t-il alors ? Quelle peut être la trame de sa fantaisie suicidaire? Dans son versant omnipotent, son fantasme de mort met en scène son désir de se débarrasser de sa carcasse d'insecte, endommagée par la poussière et la pomme pourrie de l'Autre. De cette façon, il pourrait se différencier radicalement des agresseurs qui l'ont constitué. Je propose également que son fantasme imagine une scène primitive déniée et inversée: par son suicide, Gregor engendrerait sa famille (variation sur le thème de l'autoengendrement) que sa métamorphose a détruite. Effectivement, son devenirinsecte a fait éclater le noyau familial que son devenir-mort ressouderait. Il serait paradoxalement immortel entre ses parents parce que sa mort les aurait symbiotiquement réunis. D'ailleurs, échapper à son corps, marqué d'une étrangeté insoutenable, serait la condition pour retrouver ce temps qui précède sa différenciation interdite. Il a faim «d'une nourriture inconnue à laquelle il aspire ardemment» (p. 136), nourriture qui évoque peut-être celle du sein idéal de sa préhistoire, l'objet mythique déjà perdu. Il cherche d'abord cette nourriture du côté de la vie: sa quête devient agonie. C'est alors du côté de la mort qu'il fantasme satisfaire sa quête d'indifférencié, d'une fusion que sa métamorphose a mise hors d'atteinte.

\section{Pour conclure}

La trajectoire suivie par l'essai est claire: j'ai articulé les fantasmes de suicidelimite à l'organisation de la personnalité, marquée d'un interdit de différenciation, dans laquelle ils s'inscrivent. Effectivement, d'abord dans la petite enfance et ensuite dans l'adolescence, le traumatisme des limites se répètent : la séparation de la personnalité-limite est rendue impossible par un Autre intrusif qui l'aliène à son expérience et dont l'étrangeté hostile devient constituante de son self. Les identifications projectives parentales font violence à sa différence subjective laissée vide, 
manquant à être soutenue d'un regard accueillant. Conséquemment, il est bloqué entre psychose et névrose, entre incapacité et capacité à être sujet, trop possédé par le désir d'un Autre pour que le sien puisse advenir sans risque d'un abandon punitif. Le fantasme de suicide crée alors un espace psychique paradoxal qui s'étaie sur un clivage de l'expérience de soi et du monde : il permet d'imaginer une individuation radicale - par un désengagement de l'emprise des mauvais objets internes — en même temps qu'un retour à l'indifférencié — par une fusion avec la mère idéale d'avant les origines. La métamorphose de Gregor Samsa m'a permis d'illustrer les pôles de ce dilemme qui tue la subjectivité, lorsque c'est la faute de vivre différent qui s’impose comme (fausse) alternative à la mort.

anthony bourgeault

3156, édouard-montpetit, app. 4 montréal

h3t $1 \mathrm{j} 9$

anthony.bourgeault@umontreal.ca

\section{Notes}

1. (Que Sartre dorme tranquille.)

2. Une discussion du contenu incestueux traumatique de la nouvelle aurait mérité plus amples développements. L'analyse présentée est davantage centrée sur un aspect de la relation familiale : l'intensité agressive.

\section{Bibliographie}

Bateman, A. (2004), Le narcissisme et sa relation à la violence et au suicide, in Violence et suicide, PUF, Paris. p. 186207.

Bergeret, J. (1986), Les États-limites et leurs Aménagements, in Psychologie pathologique, Masson, Paris, p. $193-$ 210.

Bollas, C. (1996), Une théorie du vrai self, in Les forces de la destinée, Calman-Lévy, Paris, p. 21-39.

Cahn R. (1988). Fonction et destin de l'espace transitionnel à l'adolescence : entre leurre et vérité, in Adolescences, Privat, Toulouse, p. 117-126.

Cahn, R. (1991), Adolescence et folie, PUF, Paris, 312 p.

Cahn, R. (1998), L'adolescent dans la psychanalyse : l'aventure de la subjectivation, PUF, Paris, 217 p.

Campbell, D. (2004), Le rôle du père dans l'état pré-suicidaire, in Violence et suicide, PUF, Paris, p. 134-151.

Deleuze, G. Guattari. F. (1975), Kafka: pour une littérature mineure, Les Éditions Minuit, Paris, 157 p.

Fonagy, P. (2004), Remarques finales, in Violence et suicide, PUF, Paris, p. 258-272.

Jeammet, P. Alléon, A.-M. Birot, E. Cremniter, D. (1994), Le fonctionnement mental de l'adolescent et du jeune adulte suicidants, in Étude psychopathologique des tentatives de suicide chez l'adolescent et le jeune adulte, PUF, Paris, p. 89-132.

Joseph Perelberg, R. (2004), La compréhension psychanalytique de la violence et du suicide: une revue de la littérature et quelques nouvelles formulations, in Violence et suicide, PUF, Paris, p. 49-99.

Kafka, F. (1989), La Métamorphose in La métamorphose et autres récits, Gallimard, Paris, p. 79-148. 


\section{2}

Filigrane, printemps 2006

Kamel, F. (1988), Le suicide de l'adolescent in Adolescences, Privat, Toulouse, p. 143-146.

Ladame, F. (1991), Les tentatives de suicide des adolescents : Pourquoi ? Comment? in Adolescence et Suicide, ESF Éditeur, Paris, p. 17-36.

Ladame, F. Ottino. J. (1993), Les paradoxes du suicide, in Adolescence, 11, 1, p. 125-136.

Le Run, J. L. (1999), L'intime et l'étranger: paradoxes de l'identité, in La figure de l'Autre, étranger, en psychopathologie clinique, L'Harmattan, Paris, p. 61-70.

Magnenat Bourquin, A. (1995), Approche psychodramatique de l'adolescent personnalité-limite : les effets d'émergence subjective, Adolescence, 25, p. 129-141.

Maltsberger, J. BUIE, D. (1980), The devices of suicide, International Review of Psycho-Analysis, 61, 7, p. 61-72.

Maltsberger, J. (2004), The descent into suicide, International Journal of Psychoanalysis, 85, 3, p. 653-668.

Masterson, J. (1975), The borderline syndrome: the role of the mother in the genesis and psychic structure of the borderline personality, International Journal of Psychoanalysis, 56, 2, p. 163-178.

Masterson, J. (1987), Resistance of the borderline patient with a false self, in Techniques of Working with Resistance. Jason Aronson, London, p. 339-361.

Manzano, J. Espaza, F. ZIilkha, N. (1999), Les scénarios narcissiques de la parentalité, PUF, 174 p.

Ottino, J. (1995), Suicide et psychopathologie : regard actuel in Adolescence et Suicide, Masson, Paris, p. 77-83.

Painchaud, G. Mongrain, N. (1986), Limites et états-limites, in Narcissisme et États-limites, Dunod, Montréal, p. 2835.

Paz, C.A. Olmos PAZ, T. (1992), Adolescence and borderline pathology, International Journal of Psychoanalysis, 73, 4, p. 739-755.

Ribas, D. (1991), La mort comme origine, une figure de l'auto-engendrement: «La jetée» de Chris Marker, Revue française de psychanalyse, 55, 5, p. 1237-1241.

Ribas, D. (1991), On tue un sujet, Revue française de psychanalyse, 55, 6, p. 1789-1792.

Richard, F. (1998), Les troubles psychiques à l'adolescence, Dunod, Paris, 113 p.

Richard, F. (2001), Le processus de subjectivation à l'adolescence, Dunod, Paris, p. 278.

Samy, M. (1991), Suicide et Adolescence: une perspective sociale et analytique, in Adolescence et Suicide, ESF Éditeur, Paris, p. 59-76.

Schashter, J. (2004), Le paradoxe du suicide : questions d'identité et de séparation, in Violence et suicide, PUF, Paris, p. $238-257$.

Shapiro, E. Zlinner, J. Shapiro, R. Berkowitz, D. (1975), The influence of family experience on borderline personality development, International Review of Psycho-Analysis, 2, 4, p. 399-411.

Thiébaut, C. (1991), La métamorphose et autres récits de Franz Kafka, Gallimard, Paris, 251 p.

Wainrib, S. (1996), Angoisse de néantisation, fascinations de l'anéantissement, Revue française de psychanalyse, 60, 1, p. $65-76$.

Winnicott, D.W. (1955), L'agressivité et ses rapports avec le développement affectif, in De la pédiatrie à la psychanalyse, Petite Bibliothèque Payot, p. 80-97.

Winnicott, D. W. (1958), La capacité d'être seul, in De la pédiatrie à la psychanalyse, Petite Bibliothèque Payot, Paris, p. 205-213.

Winnicott, D.W. (1962), L'adolescence, in De la pédiatrie à la psychanalyse. Petite Bibliothèque Payot. p. 257-266.

Winnicott, D.W. (1974), Distorsion du moi en fonction du vrai et du faux self, in Processus de maturation chez l'enfant, PUF, Paris, p. 115-131.

Winnicott, D.W. (1975), Jeu et réalité, Gallimard, 276 p. 